\title{
Downregulation of the expression of B-cell lymphoma-extra large by RNA interference induces apoptosis and enhances the radiosensitivity of non-small cell lung cancer cells
}

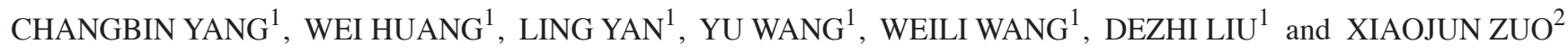 \\ Departments of ${ }^{1}$ Radiation Oncology and ${ }^{2}$ Nuclear Medicine, The Tumor Hospital of Jilin Province, \\ Changchun, Jilin 130012, P.R. China
}

Received May 13, 2014; Accepted January 27, 2015

DOI: $10.3892 / \mathrm{mmr} .2015 .3346$

\begin{abstract}
B-cell lymphoma-extra large (Bcl-xL), an important member of anti-apoptotic Bcl-2 family, is involved in tumor progression and development. The overexpression of Bcl-xL is associated with radioresistance of human malignancies. The present study aimed to investigate the inhibitory effect of small interfering RNA (siRNA) on the expression of Bcl-xL in the A549 non-small lung cancer (NSCLC) cell line, and its role in inducing the apoptosis and increasing the radiosensitivity of A549 cells. An siRNA expression vector, pSilencer4-CMVneo-short hairpin (sh)RNA, was constructed and stably transfected into A549 cells. The effects of Bcl-xL-shRNA on cell proliferation, apoptosis and the protein expression levels of associated proteins were assessed in vitro in the A549 cells. The radiosensitivity of the A549 cells was evaluated using a clonogenic cell survival assay. The results demonstrated that the sequence-specific siRNA targeting Bcl-xL efficiently and specifically downregulated the mRNA and protein expression levels of Bcl-xL. The RNA interference-mediated downregulation in the expression of $\mathrm{Bcl}-\mathrm{xL}$ inhibited cell proliferation, induced apoptosis and reduced the radioresistance of the NSCLC cells. These findings suggested that Bcl-xL may be a promising therapeutic approach for the treatment of NSCLC.
\end{abstract}

\section{Introduction}

Lung cancer is the most common cause of cancer-associated mortality and its morbidity is increasing worldwide, and $\sim 85-90 \%$ of lung cancer cases are non-small cell lung cancer (NSCLC) (1). Surgery, chemotherapy and radiation are the

Correspondence to: $\mathrm{Dr}$ Xiaojun Zuo, Department of Nuclear Medicine, The Tumor Hospital of Jilin Province, 1018 Huguang Road, Changchun, Jilin 130012, P.R. China

E-mail: zuoxiaojun510@sina.com

Key words: non-small lung cancer, B-cell lymphoma-extra large, RNA interference, radiosensitivity three major therapeutic approaches for the treatment of lung cancer. Radiotherapy is commonly used for the treatment of NSCLC, and $>50 \%$ of newly diagnosed patients with lung cancer worldwide receive radiotherapy, either alone or in combination with surgery or chemotherapy, during their treatment (2). However, the rates of complete recovery are low and long-term survival rates remain poor, since the curative potential of radiotherapy is often limited due to intrinsic radioresistance of cancer cells and systemic dose-limiting toxicity to normal tissues (3-6). Therefore, there is increasing interest in enhancing the radiosensitivity of lung cancer cells for the development of more effective and less toxic treatments.

Proteins of the B-cell lymphoma (Bcl)-2 family have been identified as key regulators of apoptosis (7). The Bcl-2 family of proteins is comprised of anti-apoptotic proteins, including Bcl-2, Bcl-extra large $(x \mathrm{~L})$ and myeloid leukemia cell differentiation protein; pro-apoptotic proteins, including Bcl-2-associated X protein (Bax); and Bcl-2-antagonist/killer and Bcl-2 homology domain 3-only proteins, including Bim, Bcl-2-associated death promoter (Bad), Phorbol-12-myristate-13-acetate-induced protein 1, p53 upregulated modulator of apoptosis and harakiri (8). The overexpression of anti-apoptotic Bcl-2 family proteins contributes to the development of cancer and to cancer cell resistance against a wide variety of anticancer agents (9-11). Bcl-xL, a major member of the anti-apoptotic Bcl-2 family, is overexpressed in NSCLC $(12,13)$. The overexpression of Bcl-xL has been observed to counteract the pro-apoptotic functions of Bax and Bad by preventing their translocation between the cytosol and the mitochondria (14). Several studies have revealed that inhibiting the expression of Bcl-xL, using antisense oligonucleotides or small interfering (si)RNA, suppresses the proliferation of and sensitizes tumor cells to chemotherapeutic agents (15-17). Varin et al demonstrated that knockdown of Bcl-xL using siRNA sensitizes two highly chemoresistant mesothelioma cell lines to treatment with cisplatin (18). Guichard et al found that short hairpin (sh) RNAs targeting Bcl-xL modulated senescence and apoptosis following exposure to $\mathrm{SN}-38$ and irinotecan in a model of colon cancer (15). Lei et al demonstrated that Bcl-xL siRNA contributed to an increase in diamminedichloroplatinum (DDP)-induced cell death in NSCLC and sensitized cells to DDP, leading to an increase the effectiveness of the drug in 
treating NSCLC (19). Notably, a previous study revealed that downregulation of Bcl-xL using siRNA increased the in vitro and in vivo radiosensitivity of colorectal cancer cells by increasing caspase-dependent apoptosis (20). These studies suggest that the protein expression of Bcl-xL is critical for tumor development, progression and resistance to therapy, including chemotherapy and radiation. However, whether the inhibition of $\mathrm{Bcl}-\mathrm{xL}$ is an effective approach to overcome the radioresistance exhibited by NSCLC remains to be elucidated. The present study aimed to examine $\mathrm{Bcl}-\mathrm{xL}$ as a therapeutic target for the treatment of human NSCLC.

The successful use of siRNA in downregulating gene expression in a number of model systems has led to several attempts to examine this methodology as a potentially therapeutic approach (21). DNA vector-based shRNA technology can achieve persistent silencing of endogenous gene expression (22). In the present study, the expression of Bcl-xL was downregulated using RNA interference (RNAi) to investigate the role of $\mathrm{Bcl}-\mathrm{xL}$ in radioresistance, and to determine the feasibility and efficacy of combination therapy, involving siRNA targeting Bcl-xL and radiotherapy, on NSCLC cells.

\section{Materials and methods}

shRNA design and plasmid construction. The cDNA sequence of Bcl-xL was obtained from GenBank (accession no. Z23115). Bcl-xL-shRNA was designed using siRNA target design finder (AmbionInc.,Austin,TX,USA) and the sequences wereas follows: Bcl-xL-shRNA, sense 5'-CAGGGACAGCATATCAGAG-3' and antisense 5'-GTCCCTGTCGTATAGTCTC-3'. The oligonucleotides were annealed and inserted into the BamHI and HindIII sites on the pSilencer4.1-CMVneo vector (Ambion Inc.), according to the manufacturer's instructions (Ambion Inc.). The pSilencer4.1-CMVneo vector contains the SV40 early promoter to provide G418 resistance in mammalian cells. The recombinant vectors were confirmed by digestion analysis using restriction endonucleases Bam HI and HindIII (Takara Bio, Inc., Dalian, China) at $37^{\circ} \mathrm{C}$ for $2 \mathrm{~h}$ and the inserted sequences were verified by DNA sequencing. A negative control vector, expressing a hairpin siRNA with limited homology to any known sequences of the human genome, was commercially available (Ambion Inc.). The shRNA vector containing the oligonucleotides encoding Bcl-xL was termed Bcl-xL-shRNA and the negative control (NC) vector was termed NC-shRNA. The purified DNA was diluted to $1 \mathrm{mg} / \mathrm{ml}$ and stored at $-20^{\circ} \mathrm{C}$ until use.

Cell culture and transfection. The A549 human NSCLC cell line was purchased from Cell Bank of Type Culture Collection of Chinese Academy of Sciences (Shanghai, China). The A549 cells were cultured in RPMI-1640 medium (Invitrogen Life Technologies, Carlsbad, CA, USA) supplemented with heat-inactivated $10 \%$ fetal bovine serum (Biochrom AG, Berlin, Germany), $100 \mathrm{U} / \mathrm{ml}$ penicillin and $100 \mathrm{mg} / \mathrm{ml}$ streptomycin (Sigma-Aldrich, St. Louis, MO, USA) at $37^{\circ} \mathrm{C}$ in a humidified atmosphere containing $5 \% \mathrm{CO}_{2}$.

The A549 cells were then seeded into 6-well plates at a density of $2 \times 10^{4}$ cells/well and cultured overnight to $80-90 \%$ confluence prior to transfection. Transfection was performed using Lipofectamine Plus (Invitrogen Life Technologies), and the ratio of the plasmids to transfection reagent was $1 \mathrm{mg}: 2 \mathrm{ml}$. The cells were transfected with either the Bcl-xL-shRNA or NC-shRNA plasmids, according to the manufacturer's instructions. G418 (800 $\mu \mathrm{g} / \mathrm{ml}$; Sigma-Aldrich) was used to screen for stably transfected clones. The stable transfectants were termed A549/Bcl-xL-shRNA and A549/NC-shRNA.

Reverse transcription-quantitative polymerase chain reaction (RT-qPCR). The transfected and non-tranfected cells were collected and washed with phosphate-buffered saline (PBS; Sigma-Aldrich). The total RNA was extracted from the cells using TRIzol reagent (Invitrogen Life Technologies), according to the manufacturer's instructions. Moloney Murine Leukemia Virus reverse transcriptase (Fermentas, Waltham, MA, USA) was used to amplify the cDNA, according to the manufacturer's instructions. The RT-qPCR assays were performed using SYBR TAQ real-time kits (Takara Bio, Inc., Otsu, Japan) and RT-PCR amplification equipment (ABI PRISM 7900HT; Applied Biosystems, Foster City, CA, USA). The primer sequences used for qPCR were as follows: Bcl-xL, sense 5'-CGTGGAAAGCGTAGACAAGGA-3' and antisense 5'-ATTCAGGTAAGTGGCCATCCAA-3' and GAPDH, sense 5'-TGTGGGCATCAATGGATTTGG-3' and antisense 5'-ACACCATGTATTCCGGGTCAAT-3'. The PCR conditions were as follows: Predenaturation at $94^{\circ} \mathrm{C}$ for $5 \mathrm{~min}$, followed by 40 cycles of denaturation at $94^{\circ} \mathrm{C}$ for $10 \mathrm{sec}$, annealing/extension at $60^{\circ} \mathrm{C}$ for $15 \mathrm{sec}$ and final extension at $72^{\circ} \mathrm{C}$ for $10 \mathrm{~min}$. The specificity of the amplification was confirmed using melting curve analysis. The expression of target genes were normalized against the expression of GAPDH. The fold-change was calculated, as described previously (23), and the data are presented as the fold-change in expression relative to the untransfected controls.

Western blotting. The cells were homogenized in lysis buffer (Sigma-Aldrich) containing $50 \mathrm{mmol} / \mathrm{l}$ Tris-HCl, $5 \mathrm{mmol} / \mathrm{l}$ EDTA, $150 \mathrm{mmol} / 1 \mathrm{NaCl}, 1 \%$ sodium deoxycholate, $500 \mu \mathrm{mol} / 1 \mathrm{Na}_{3} \mathrm{VO}_{4}, 0.5 \%$ Triton X-100, $10 \mu \mathrm{mol} / 1$ 4-(2-aminoethyl) benzenesulfonyl fluoride hydrochloride (AEBSF) and $10 \mathrm{mmol} / 1 \mathrm{NaF}$, on ice for $30 \mathrm{~min}$. The homogenates were subsequently centrifuged at $12,000 \mathrm{x} \mathrm{g}$ at $4^{\circ} \mathrm{C}$ for $15 \mathrm{~min}$, the supernatants, containing the total cellular protein, were collected and the protein concentration was determined using a Bicinchoninic Acid Assay kit (Sigma-Aldrich). Equal quantities of protein lysate $(50 \mu \mathrm{g})$ were electrophoretically separated on 10 or $8 \%$ sodium dodecyl sulfatepolyacrylamide gels, transferred onto polyvinylidene difluoride membranes (Millipore, Bedford, MA, USA) and were blocked in $3 \%$ bovine serum albumin for $2 \mathrm{~h}$. Following blocking, the membranes were incubated with the following antibodies: Mouse anti-Bcl-xL monoclonal antibody (1:1,500; sc-271121), mouse anti-caspase-3 polyclonal antibody $(1: 2,000 ;$ sc-7272) and mouse anti-caspase- 8 polyclonal antibody $(1: 3,000 ;$ sc-56070), which were all purchased from Santa Cruz Biotechnology, Inc (Santa Cruz, CA, USA), as well as mouse anti-poly(ADP-ribose) polymerase (PARP) polyclonal antibody (1:1,000; \#9544; Cell Signaling Technology, Inc., Beverly, MA, USA) and mouse monoclonal anti- $\beta$-actin (1:5,000; A2228; Sigma-Aldrich), overnight at $4^{\circ} \mathrm{C}$. The membranes were subsequently incubated for $2 \mathrm{~h}$ at $37^{\circ} \mathrm{C}$ with horseradish peroxidase-conjugated anti-mouse secondary 
A

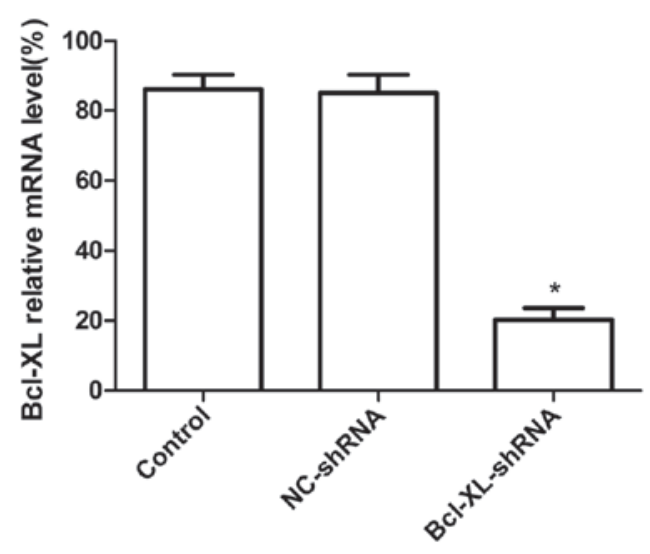

B

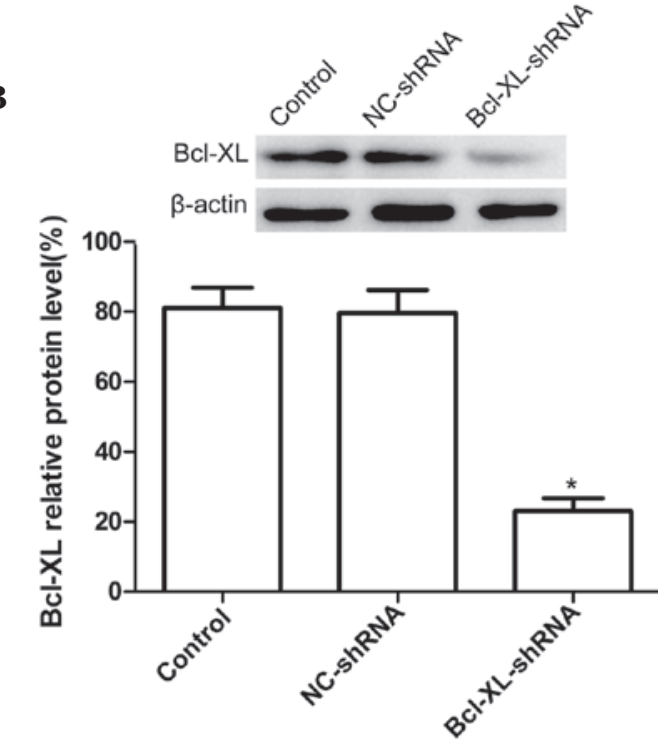

Figure 1. Detection of the mRNA and protein expression of Bcl-xL by RT-qPCR and western blotting, respectively. (A) RT-qPCR analysis of the mRNA expression of Bcl-xL in the A549 cells $48 \mathrm{~h}$ after transfection with Bcl-xL-shRNA. (B) Western blot analysis of the protein expression of Bcl-xL in the A549 cells $48 \mathrm{~h}$ after transfection with Bcl-xL-shRNA. $\beta$-actin was used as an internal control. Data are expressed as the mean \pm standard deviation ( $\mathrm{P}<0.05$, vs. untransfected control). sh, short-hairpin; NC, negative control; Bcl-xL, B-cell lymphoma-extra large; RT-qPCR, reverse transcription-quantitative polymerase chain reaction.

antibody (Santa Cruz Biotechnology, Inc.). $\beta$-actin was used as a loading control. The bound antibodies were detected using an enhanced chemilluminescence kit (Santa Cruz Biotechnology, Inc.). Densitometric analysis was performed using Quantity One image analysis software (Bio-Rad Laboratories, Hercules, CA, USA).

Cell proliferation assay. The cell viabilities of the untransfected, stably transfected A549/Bcl-xL-shRNA and A549/NC-shRNA A549 cells were measured using a 3-(4, 5-dimethylthazol-2-yl)-2, 5- diphenyl tetrazolium bromide (MTT) assay (Sigma-Aldrich). Briefly, the A549 cells were seeded into seven 96-well plates at a density of $4 \times 10^{3}$ cells/well, with eight wells per group/subgroup. Following $48 \mathrm{~h}$ culture, $200 \mu \mathrm{l}$ MTT $(5 \mathrm{mg} / \mathrm{ml})$ was added to each well, followed by incubation at $37^{\circ} \mathrm{C}$ for $4 \mathrm{~h}$. The supernatant was then removed and $200 \mu \mathrm{l}$ dimethyl sulfoxide was added to each well, followed by agitation for $10 \mathrm{~min}$. The optical densities were determined using a Versamax microplate reader (Molecular Devices, LLC., Sunnyvale, CA, USA) at $490 \mathrm{~nm}$, and the growth inhibition was calculated as follows: Inhibition rate $(\%)=[1$-(average absorbance of experimental group/average absorbance of blank control group)]x100\%.

Analysis of apoptosis. Cell apoptosis was identified by fluorescence staining using acridine orange (AO) and ethidium bromide (EB) from Molecular Probes (Eugene, OR, USA). For the morphological examination of apoptosis, the untransfected, stably transfected A549/Bcl-xL-shRNA and A549/NC-shRNA A549 cells $\left(5 \times 10^{3} /\right.$ well) were seeded into a separate 24-well microplate for $48 \mathrm{~h}$, washed three times with PBS and mixed with an identical volume of dual $\mathrm{AO} / \mathrm{EB}$ solution $(100 \mu \mathrm{g} / \mathrm{ml})$. The final volume $(200 \mu \mathrm{l})$ was observed using a CKX41 fluorescence microscope at x20 magnification (Olympus, Tokyo, Japan). For quantification, five random fields were selected and at least 300 cells were quantified in each field. All experiments were performed in triplicate. At the molecular level, the protein expression levels of PARP, caspase- 3 and caspase- 8 were assessed by western blotting, as described above, an additional indicator of apoptosis.

Clonogenic cell survival assay. The untransfected or stably transfected A549/Bcl-xL-shRNA and A549/NC-shRNA A549 cells were seeded seperately at a density of $5 \times 10^{3}$ cells/well into each well of 96-well plates in triplicate. Following culture for $72 \mathrm{~h}$, the cells were trypsinized and cells ( $1 \times 10^{4} /$ well) were seeded into six-well plates and allowed to attach for $6 \mathrm{~h}$ at $37^{\circ} \mathrm{C}$ in a humidified atmosphere containing $5 \% \mathrm{CO}_{2}$. The cells were then irradiated with different doses $(0,2,4,6$, and $8 \mathrm{~Gy})$ of $6 \mathrm{MV}$ X-ray radiation using a 23EX accelerator (Varian Medical Systems,. Inc., Palo Alto, CA, USA) at room temperature, and were subsequently incubated for 14 days. The colonies were stained with crystal violet and the number of colonies containing $>50$ cells were quantified. The plating efficiency was calculated as follows: Plating efficiency $(\%)=$ (colony number / total cells seeded) x $100 \%$. All experiments were performed in triplicate. The cell survival fraction was determined and a cell survival curve was produced.

Statistical analysis. All experiments were performed in triplicate as independent experiments. The data are expressed as the mean \pm standard deviation. Comparisons between two samples were calculated using Student's t-test and comparisons of $>2$ groups were calculated using one-way analysis of variance followed by a Tukey's post hoc test using Graphpad Prism 6.0 software (San Diego, CA, USA). $\mathrm{P}<0.05$ was considered to indicate a statistically significant difference.

\section{Results}

Specific downregulation of the expression of Bcl-xL by $B c l-x L-s h R N A$. The mRNA and protein expression levels of $\mathrm{Bcl}-\mathrm{xL}$ in the NSCLC cells were analyzed by RT-qPCR and 
Table I. Plating efficiency at different radiation doses.

\begin{tabular}{lccccr}
\hline & \multicolumn{5}{c}{ Plating efficiency (\%) } \\
\cline { 2 - 6 } Cell group & 0 Gy & 2 Gy & 4 Gy & 6 Gy & 8 Gy \\
\hline A549 cell & $96.45 \pm 2.12$ & $82.45 \pm 2.08$ & $66.24 \pm 1.48$ & $44.34 \pm 1.67$ & $21.12 \pm 1.07$ \\
A549/NC-shRNA & $97.67 \pm 2.41$ & $80.45 \pm 1.33$ & $64.38 \pm 0.78$ & $43.45 \pm 1.88$ & $19.89 \pm 0.84$ \\
A549/Bcl-xL-shRNA & $89.89 \pm 1.35$ & $54.23 \pm 0.89^{\mathrm{a}}$ & $31.23 \pm 0.56^{\mathrm{a}}$ & $9.89 \pm 0.45^{\mathrm{a}}$ & $3.35 \pm 0.38^{\mathrm{a}}$
\end{tabular}

Data are expressed as the mean \pm standard deviation ( $\left({ }^{\mathrm{P}}<0.05\right.$, vs. control). Gy, Grays; NC, negative control; Bcl-xL, B-cell lymphoma-extra large; sh, short hairpin.

western blotting. As shown in Fig. 1A, the mRNA expression of Bcl-xL in the Bcl-xL-shRNA group was significantly decreased compared with the untransfected control group and the NC-shRNA group $(\mathrm{P}<0.05)$. No significant difference was observed between the NC-shRNA group and the control group. Additionally, the protein expression level was significantly reduced in the Bcl-xL-shRNA group compared with the control and NC-shRNA groups $(\mathrm{P}<0.05$; Fig. 1B). No significant change in the protein expression levels of $\mathrm{Bcl}-\mathrm{xL}$ was observed between the NC-shRNA group and control group $(\mathrm{P}>0.05)$. These results demonstrated that the expression levels of Bcl-xL in the A549 cells were downregulated, specifically and effectively, by Bcl-xL-shRNA.

Effect of Bcl-xL-shRNA on A549 cell proliferation. Using $\mathrm{Bcl}-\mathrm{xL}$-shRNA, the effects of downregulation of Bcl-xL on tumor cell proliferation were examined in vitro using an MTT assay (Fig. 2). The results demonstrated that transfection of the A549 cells with Bcl-xL-shRNA significantly inhibited cell proliferation compared with the control and NC-shRNA groups $(\mathrm{P}<0.01)$.

Effect of XIAP-shRNA on the apoptosis of A549 cells. To further investigate the effect of the shRNA-mediated downregulation of XIAP, on cell apoptosis in the A549 cells the, A549/Bcl-xL-shRNA and A549/NC-shRNA stably transfected cells were collected and stained with AO/EB. The results demonstrated that cells transfected with $\mathrm{Bcl}-\mathrm{xL}$ siRNA underwent typical apoptotic morphological changes of nuclear and cytoplasmic condensation, loss of cell volume and nuclear fragmentation. By contrast, the untransfected and NC-shRNA transfected cells exhibited no apoptotic characteristics (Fig. 3A). Statistical analysis revealed that the A549 cells transfected with Bcl-xL-shRNA significantly induced cell apoptosis compared with the untransfected and the NC-shRNA cells $(\mathrm{P}<0.01$; Fig. 3B). Therefore, Bcl-xL-shRNA significantly accelerated the apoptosis of A549 cells.

Preliminary mechanisms underlying Bcl-xL-regulated cell apoptosis. To examine the mechanism underlying the induction of cell apoptosis, the expression levels of PARP, caspase- 3 and caspase- 8 in the A549 cells were determined by western blotting, following treatment with Bcl-xL-shRNA or NC-shRNA. As shown in Fig. 4, the expression levels of caspase-3, caspase- 8 and PARP were markedly increased

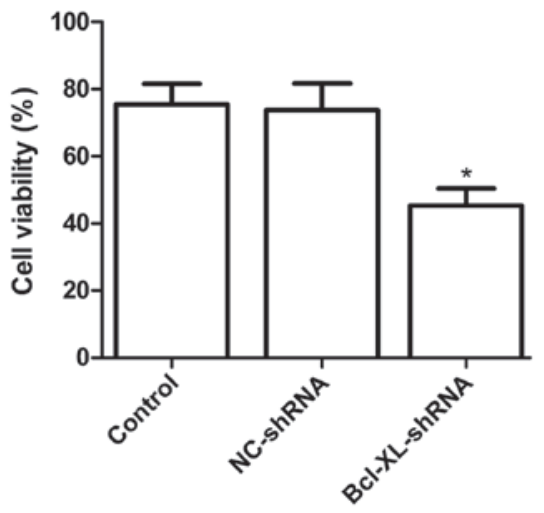

Figure 2. Bcl-xL-shRNA reduces the viability of A549 cells. The viabilities of the untransfected and stably transfected A549 cells were evaluated using an MTT assay. The viability of the Bcl-xL-shRNA cells was significantly decreased compared with the untransfected and NC-shRNA cells. Data are expressed as the mean \pm standard deviation ( ${ }^{*} \mathrm{P}<0.05$, vs. untransfected control) $\mathrm{NC}$, negative control; Bcl-xL, B-cell lymphoma-extra large; sh, short hairpin.

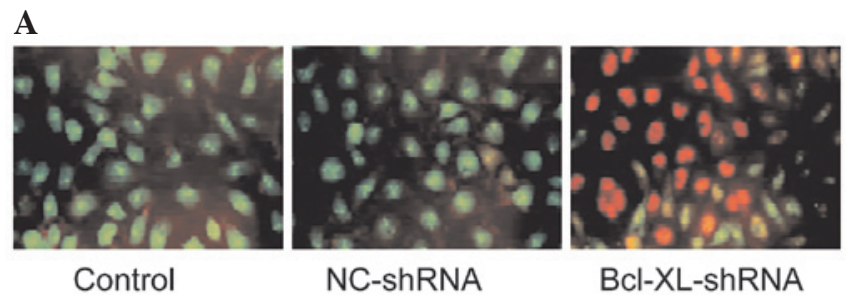

B

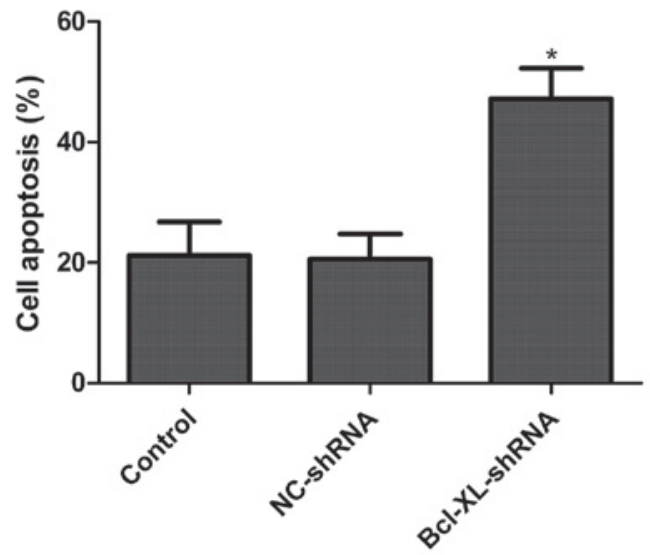

Figure 3. Bcl-xL-shRNA affects the apoptosis of A549 cells. (A) Apoptotic rates of the untranfected and stably transfected A549 cells were evaluated by acridine orange/ethidium bromide staining. (B) Downregulation of the expression of Bcl-xL by Bcl-xL-shRNA induced cell apoptosis. The data are expressed as the mean \pm standard deviation ( ${ }^{*} \mathrm{P}<0.05$, vs. untransfected contol). $\mathrm{NC}$, negative control; Bcl-xL, B-cell lymphoma-extra large; sh, short hairpin. 
A
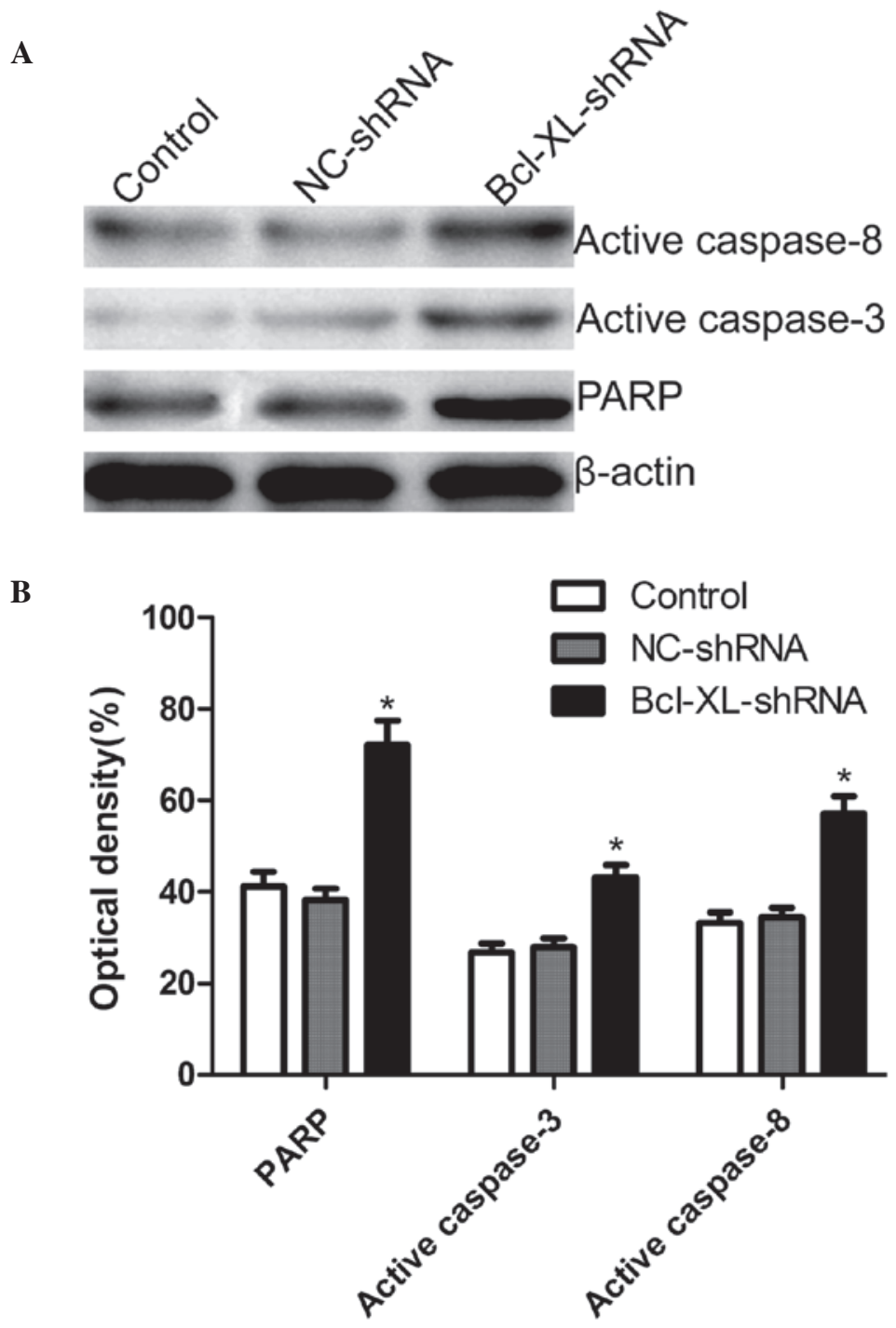

Figure 4. Bcl-xL-shRNA affects the expression levels of apoptosis-associated proteins, determined by western blotting. (A) Expression levels of PARP, caspase- 3 and caspase- 8 were determined by western blotting. (B) Relative quantification of active PARP, caspase-3 and caspase- 8 proteins by densitometric analysis. Data are expressed as the mean \pm standard deviation ( $(\mathrm{P}<0.05$, vs. untrasfected control). NC, negative control; Bcl-xL, B-cell lymphoma-extra large; sh, short hairpin; PARP, poly(ADP ribose) polymerase.

in the cells transfected with Bcl-xL-shRNA compared with those in the untransfected and the NC-shRNA-transfected cells.

Effect of Bcl-xL-shRNA on the radiosensitivity of A549 cells. To investigate the effect of Bcl-xL-shRNA on the radiosensitivity of A549 cells, clonogenic cell survival assays were performed. As shown in Table I, the plating efficiencies of the A549 cells transfected with Bcl-xL-shRNA cells at the same dose of radiation were significantly decreased compared with the control cells and the cells transfected with NC-shRNA $(\mathrm{P}<0.05)$. The cell survival curve revealed a marked decreased in the survival of the cells in the Bcl-xL-shRNA group compared with that observed in the untransfected A549 cells and the NC-shRNA group $(\mathrm{P}<0.05$; Fig. 5). No significant difference was observed in the radiosensitivity of the untransfected cells and NC-shRNA cells. These results demonstrated that downregulatiion in the expression of $\mathrm{Bcl}-\mathrm{xL}$ led to enhanced radiosensitivity in the NSCLC cells.

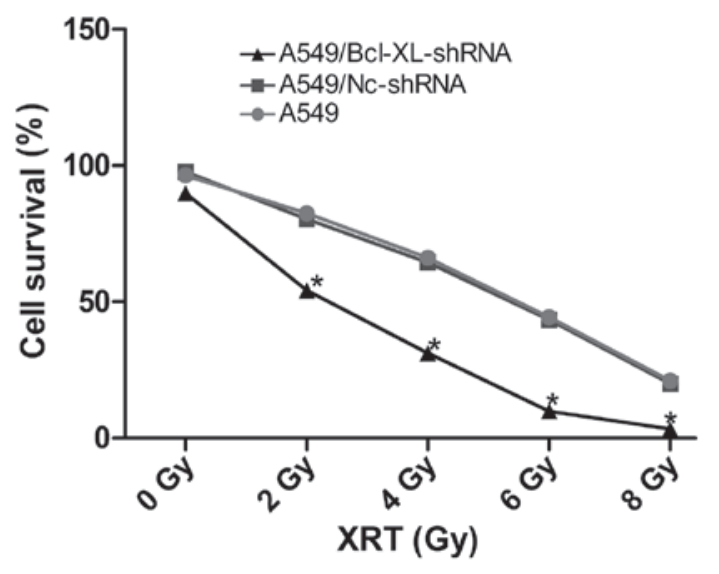

Figure 5. Effect of radiation on the survival of stably transfected A549 cells. The untransfected and stably transfected NC-shRNA and Bcl-xL-shRNA A549 cells were trypsinized and seeded for cell clonogenic assays. After $6 \mathrm{~h}$, the attached cells were irradiated and the colonies were stained with crystal violet and counted following incubation for 14 days. A cell survival curve was produced. NC, negative control; Bcl-xL, B-cell lymphoma-extra large; sh, short hairpin; Gy, grays; XRT, radiation dose. 


\section{Discussion}

NSCLC patients with non-resectable stage III or medically inoperable disease account for $\sim 40 \%$ of all patients diagnosed with NSCLC (24). Radiation therapy is important in achieving local control of the tumor and in the relief of symptoms resulting from metastatic disease, therefore, radiotherapy is important in the management of NSCLC (25). However, partial lung cancer cell resistance to radiotherapy affects the therapeutic effects, and the 5-year survival rate of patients receiving radiotherapy alone is $5-10 \%$ (26). Local recurrence occurs in $80 \%$ of patients and metastasis occurs in $60 \%$ of patients (27), therefore, overcoming the resistance of NSCLC to radiotherapy remains a major challenge and requires further investigation to identify an effective radiosensitizer, which enhances tumor radiosensitivity with minimal negative effects on normal tissues.

The Bcl-2 family comprises a group of structurally associated proteins, which are fundamental in the regulation of the intrinsic pathway by controlling mitochondrial membrane permeability and the release of the the pro-apoptotic factor, cytochrome $c$ (28). Therefore, the Bcl-2 family are key regulators of apoptosis and are important in regulating cell apoptosis (7). Bcl-xL is an important member of the anti-apoptotic Bcl-2 family, which has been reported to be important in tumor progression, development and chemoresistance (15-19). Several studies have demonstrated that Bcl-xL is involved in tumor apoptosis and is important in radioresistance in several types of tumor (20,29-31). Yang et al demonstrated that the downregulation of Bcl-xL by adenovirus-mediated shRNA increases the in vitro and in vivo radiosensitivity of colorectal cancer cells by increasing caspase-dependent apoptosis (20). Streffer et al revealed that the $\mathrm{Bcl}-\mathrm{xL}$ and $\mathrm{BAX}$ proteins modulate radiosensitivity in human glioma cells, and that targeting alterations in $\mathrm{Bcl}-2$ family proteins, including the expression of $\mathrm{Bcl}-2$, may be a promising therapeutic approach to improve the efficacy of radiotherapy for gliomas (29). Masui et al demonstrated that the antisense oligonucleotide against Bcl-xL inhibits cell proliferation and increases the radiosensitivity of pancreatic cancer (30). Wang et al revealed that downregulation of Bcl-xL by siRNA increases the sensitivity of prostate cancer cells to radiation (31). However, there are no reports, to the best of our knowledge, on the association between the expression of Bcl-xL and radiosensitivity of human NSCLC cells. The present study examined changes in the radiosensitivity of A549 cells with downregulated expression of Bcl-xL using clonogenic survival assays. The results revealed that downregulation of the expression of $\mathrm{Bcl}-\mathrm{xL}$ increased cell radiosensitization, which was consistent with previous studies (20,29-31).

Caspases are important in apoptosis triggered by various pro-apoptotic signals (32). A previous study demonstrated that $\mathrm{Bcl}-\mathrm{xL}$ siRNA triggers a decrease in the protein expression of Bcl-xL and the activation of procaspase-3, followed by the cleavage of PARP, in lung cancer (19). In addition, Yang et al reported that silencing of Bcl-xL increases the activities of caspase- 3 and caspase- 8 in colorectal cancer cells (20). Consistent with this study, the present study demonstrated that downregulation Bcl-xL using shRNA activated PARP, caspase- 3 and caspase- 8 and induced cell apoptosis in the
NSCLC cells. These results suggested that the downregulation of Bcl-xL induced apoptosis in the tumor cells by increasing caspase activity.

RNAi using double stranded siRNA molecules of 20-25 nucleotides is a powerful method for preventing the expression of a particular gene, with high efficiency, high specificity and low toxicity (33). This technology is widely used to investigate gene function, cancer and viral disease therapy $(33,34)$. To examine the possibility of $\mathrm{Bcl}-\mathrm{xL}$ as an effective therapeutic target, the present study used an RNAi method to silence the endogenous expression of $\mathrm{Bcl}-\mathrm{xL}$ in the A549 NSCLC cell line and analyzed the phenotypic changes in the stable transfectants. Stable downregulation in the expression of Bcl-xL by DNA vector-based shRNA was observed in the A549 cells, which inhibited proliferation, induced apoptosis and reduced the radioresistancse of the NSCLC cells. These results suggested that $\mathrm{Bcl}-\mathrm{xL}$ may be a potential therapeutic target for the treatment of human NSCLC.

\section{Acknowledgements}

This study was supported by the Science and Technology Research and Innovation Team, funded by Jilin province (no. JL2011538).

\section{References}

1. Jemal A, Bray F, Center MM, Ferlay J, Ward E and Forman D: Global cancer statistics. CA Cancer J Clin 61: 69-90, 2011.

2. Nygren P, Glimelius B and SBU-group. Swedish Council on Technology Assessment in Health Care: The Swedish Council on Technology Assessment in Health Care (SBU) report on Cancer Chemotherapy - Project objectives, the working process, key definitions and general aspects on cancer trial methodology and interpretation. Acta Oncol 40: 155-165, 2001.

3. Impicciatore G, Sancilio S, Miscia S and Di Pietro R: Nutlins and ionizing radiation in cancer therapy. Curr Pharm Des 16: 1427-1442, 2010

4. Zhang S, Wang L, Liu H, Zhao G and Ming L: Enhancement of recombinant myricetin on the radiosensitivity of lung cancer A549 and H1299 cells. Diagn Pathol 9: 68, 2014.

5. Dumont F, Altmeyer A and Bischoff P: Radiosensitising agents for the radiotherapy of cancer: novel molecularly targeted approaches. Expert Opin Ther Pat 19: 775-799, 2009.

6. Bischoff P, Altmeyer A and Dumont F: Radiosensitising agents for the radiotherapy of cancer: advances in traditional and hypoxia targeted radiosensitisers. Expert Opin Ther Pat 19: 643-662, 2009.

7. Adams JM and Cory S: The Bcl-2 protein family: arbiters of cell survival. Science 281: 1322-1326, 1998.

8. Walensky LD: BCL-2 in the crosshairs: tipping the balance of life and death. Cell Death Differ 13: 1339-1350, 2006.

9. Yang TM, Barbone D, Fennell DA and Broaddus VC: Bcl-2 family proteins contribute to apoptotic resistance in lung cancer multicellular spheroids. Am J Respir Cell Mol Biol 41: 14-23, 2009.

10. Yip KW and Reed JC: Bcl-2 family proteins and cancer. Oncogene 27: 6398-6406, 2008.

11. Kirkin V, Joos S and Zörnig M: The role of Bcl-2 family members in tumorigenesis. Biochim Biophys Acta 1644: 229-249, 2004.

12. Soini Y, Kinnula V, Kaarteenaho-Wiik R, Kurttila E, Linnainmaa K and Pääkko P: Apoptosis and expression of apoptosis regulating proteins bcl-2, mcl-1, bcl-X, and bax in malignant mesothelioma. Clin Cancer Res 5: 3508-3515, 1999

13. Karczmarek-Borowska B, Filip A, Wojcierowski J, et al: Estimation of prognostic value of Bcl-xL gene expression in non-small cell lung cancer. Lung Cancer 51: 61-69, 2006.

14. Gottlieb E, Vander Heiden MG and Thompson CB: Bcl-x(L) prevents the initial decrease in mitochondrial membrane potential and subsequent reactive oxygen species production during tumor necrosis factor alpha-induced apoptosis. Mol Cell Biol 20: 5680-5689, 2000. 
15. Guichard SM, Hua ML, Kang P, Macpherson JS and Jodrell DI: Short hairpin RNAs targeting Bcl-xL modulate senescence and apoptosis following SN-38 and irinotecan exposure in a colon cancer model. Cancer Chemother Pharmacol 60: 651-660, 2007.

16. Zhu H, Guo W, Zhang L, et al: Bcl-xL small interfering RNA suppresses the proliferation of 5-fluorouracil-resistant human colon cancer cells. Mol Cancer Ther 4: 451-456, 2005.

17. Nita ME, Ono-Nita SK, Tsuno N, et al: Bcl-X(L) antisense sensitizes human colon cancer cell line to 5-fluorouracil. Jpn J Cancer Res 91: 825-832, 2000.

18. Varin E, Denoyelle C, Brotin E, et al: Downregulation of Bcl-xL and Mcl-1 is sufficient to induce cell death in mesothelioma cells highly refractory to conventional chemotherapy. Carcinogenesis 31: 984-993, 2010.

19. Lei X, Huang Z, Zhong M, Zhu B, Tang S and Liao D: Bcl-xL small interfering RNA sensitizes cisplatin-resistant human lung adenocarcinoma cells. Acta Biochim Biophys Sin (Shanghai) 39 344-350, 2007.

20. Yang J, Sun M, Zhang A, Lv C, De W and Wang Z: Adenovirus-mediated siRNA targeting Bcl-xL inhibits proliferation, reduces invasion and enhances radiosensitivity of human colorectal cancer cells. World J Surg Oncol 9: 117, 2011.

21. Uprichard SL: The therapeutic potential of RNA interference. FEBS Lett 579: 5996-6007, 2005.

22. Sui G, Soohoo C, Affar el B, et al: A DNA vector-based RNA technology to suppress gene expression in mammalian cells. Proc Natl Acad Sci USA 99: 5515-5520, 2002.

23. Pfaffl MW: A new mathematical model for relative quantification in real-time RT-PCR. Nucleic Acids Res 29: e45, 2001.

24. Chetty C, Bhoopathi P, Rao JS and Lakka SS: Inhibition of matrix metalloproteinase- 2 enhances radiosensitivity by abrogating radiation-induced FoxM1-mediated G2/M arrest in A549 lung cancer cells. Int J Cancer 124: 2468-2477, 2009.
25. Baumann M, Stamatis G and Thomas M: Therapy of localized non-small cell lung cancer (take home messages). Lung Cancer 33 (Suppl 1): S47-S49, 2001.

26. Duchesne GM: Fundamental bases of combined therapy in lung cancer: cell resistance to chemotherapy and radiotherapy. Lung Cancer 10 (Suppl 1): S67-S72, 1994.

27. Gressen EL and Curran WJ: Hyperfractionated radiotherapy for lung cancer. Curr Oncol Rep 2: 71-75, 2000.

28. Llambi F and Green DR: Apoptosis and oncogenesis: give and take in the BCL-2 family. Curr Opin Genet Dev 21: 12-20, 2011.

29. Streffer JR, Rimner A, Rieger J, Naumann U, Rodemann HP and Weller M: BCL-2 family proteins modulate radiosensitivity in human malignant glioma cells. J Neurooncol 56: 43-49, 2002

30. Masui T, Hosotani R, Ito D, et al: Bcl-xL antisense oligonucleotides coupled with antennapedia enhances radiation-induced apoptosis in pancreatic cancer. Surgery 140: 149-160, 2006.

31. Wang R, Lin F, Wang X, et al: Suppression of Bcl-xL expression by a novel tumor-specific RNA interference system inhibits proliferation and enhances radiosensitivity in prostatic carcinoma cells. Cancer Chemother Pharmacol 61: 943-952, 2008.

32. Lei XY, Zhong M, Feng LF, Zhu BY, Tang SS and Liao DF: Bcl-xL small interfering RNA enhances sensitivity of Hepg2 hepatocellular carcinoma cells to 5-fluorouracil and hydroxycamptothecin. Acta Biochim Biophys Sin (Shanghai) 38: 704-710, 2006.

33. Zhang J and Hua ZC: Targeted gene silencing by small interfering RNA-based knock-down technology. Curr Pharm Biotechnol 5: $1-7,2004$

34. Matsuyama Y, Yamayoshi A, Kobori A and Murakami A: Functional regulation of RNA-induced silencing complex by photoreactive oligonucleotides. Bioorg Med Chem 22: 1003-1007, 2014 